\title{
Tidal dwarf galaxies as a test of fundamental physics
}

\author{
G. Gentile ${ }^{1}$, B. Famaey ${ }^{2}$, F. Combes ${ }^{3}$, P. Kroupa ${ }^{4}$, H. S. Zhao ${ }^{5}$, and O. Tiret ${ }^{3}$ \\ 1 University of New Mexico, Department of Physics and Astronomy, 800 Yale Blvd NE, Albuquerque, NM 87131, USA \\ e-mail: ggentile@unm.edu \\ 2 Institut d'Astronomie et d'Astrophysique, Université Libre de Bruxelles, CP 226, Boulevard du Triomphe, 1050 Bruxelles, Belgium \\ 3 LERMA, Observatoire de Paris, 61 Av. de l'Observatoire, 75014 Paris, France \\ 4 Argelander-Institut für Astronomie (AIfA), Universität Bonn, Auf dem Hügel 71, 53121 Bonn, Germany \\ 5 SUPA, School of Physics and Astronomy, University of St. Andrews, KY16 9SS, Fife, UK
}

Received 13 June 2007 / Accepted 11 July 2007

\section{ABSTRACT}

\begin{abstract}
Within the cold dark matter (CDM) framework tidal dwarf galaxies (TDGs) cannot contain dark matter, so the recent results by Bournaud et al. (2007, Science, 316, 1166) that 3 rotating TDGs do show significant evidence for being dark matter dominated is inconsistent with the current concordance cosmological theory unless yet another dark matter component is postulated. We confirm that the TDG rotation curves are consistent with Newtonian dynamics only if either an additional dark matter component is postulated, or if all 3 TDGs happen to be viewed nearly edge-on, which is unlikely given the geometry of the tidal debris. We also find that the observed rotation curves are very naturally explained without any free parameters within the modified Newtonian dynamics (MOND) framework if inclinations are adopted as derived by Bournaud et al. We explore different inclination angles and two different assumptions about the external field effect. The results do not change significantly, and we conclude therefore that Newtonian dynamics has severe problems while MOND does exceedingly well in explaining the observed rotation curves of the 3 TDGs studied by Bournaud et al.
\end{abstract}

Key words. gravitation - cosmology: dark matter - galaxies: dwarf - galaxies: kinematics and dynamics

\section{Introduction}

Dwarf irregular galaxies have the tendency to form during galaxy interactions within the extended tidal tails, and are therefore dubbed Tidal Dwarf Galaxies (TDG, Mirabel et al. 1992; Duc et al. 2000; Braine et al. 2000). Tidal tails transport the angular momentum and energy away from the merging subsystems which build up the larger galaxies we see today, and gravitational instabilities within the tidal tails lead to local collapse and star formation. Observations of local interacting galaxies have shown the formation of TDGs to be quite common, in some cases dozens of condensations are seen in the tidal tails (Weilbacher et al. 2000), with a few ones having a mass typical of dwarf galaxies (Braine et al. 2000). Based on a sample of 6 local interacting galaxies, Delgado-Donate et al. (2003) estimate that at most a few long-lived TDGs forms per merger. Monreal-Ibero et al. (2007) studied in detail the stability of external star forming regions in ULIRGs, and concluded that they are good TDG candidates. The relation in terms of evolution beween TDGs and the other kinds of dwarf galaxies is presently a matter of debate (see, e.g., Duc \& Mirabel 1998; Okazaki \& Taniguchi 2000; Bournaud \& Duc 2006; Metz \& Kroupa 2007). In particular, Okazaki \& Taniguchi (2000) claim that conservative assumptions about TDG production within a hierarchical CDM structure formation framework imply that all $\mathrm{dE}$ galaxies may be TDGs leaving little or no room for traditional darkmatter filled dwarf galaxies (but see Bournaud \& Duc 2006). On the other hand, Skillman \& Bender (1995) cast some doubt on the idea that all dwarf ellipticals originate from dwarf irregulars after their gas has been blown away.
In any case, in the Cold Dark Matter (CDM) scenario, TDGs should be nearly devoid of dark matter (Barnes \& Hernquist 1992), contrary to other kinds of dwarf galaxies.

In a recent paper, Bournaud et al. (2007, hereafter B07) analyse the rotation curves of 3 TDGs belonging to the NGC 5291 system. They find evidence for a mass discrepancy that is unexpected within the CDM framework, and they put forward the hypothesis of baryonic dark matter to explain the observations. Here we investigate the possibility that the observations can be explained within the framework of MOND (Modified Newtonian Dynamics, Milgrom 1983), without the need for baryonic dark matter.

In general, disk galaxies' rotation curves do not decrease in the outer parts as would be expected from the visible matter distribution. In the standard picture, this is explained by dark matter halos around galaxies. However, MOND is an alternative explanation where our understanding of gravity (or inertia) is changed, rather than our understanding of the matter content of the Universe. Milgrom (1983) postulated that for gravitational accelerations below $a_{0} \approx 10^{-8} \mathrm{~cm} \mathrm{~s}^{-2}$ the effective gravitational attraction approaches $\left(g_{\mathrm{N}} a_{0}\right)^{1 / 2}$ where $g_{\mathrm{N}}$ is the usual Newtonian gravitational field. MOND leads to remarkable fits of galactic kinematics over 5 decades in mass (Sanders \& McGaugh 2002) ranging from tiny dwarfs (e.g., Gentile et al. 2007) through our own Milky Way (Famaey \& Binney 2005; Famaey et al. 2007) and early-type spirals (e.g., Sanders \& Noordermeer 2007), to massive ellipticals (Milgrom \& Sanders 2003), without resorting to galactic dark matter. However, possible problems for MOND on galactic scales include the kinematics of Local Group dwarf spheroidals (Łokas et al. 2006) and galaxy merging timescales (Nipoti et al. 2007). 
TDGs are thus objects on galactic scales on which to test the MOND paradigm ${ }^{1}$. Here we take into account uncertainties on the external field effect (EFE) and the galaxies' inclinations to show that the MOND paradigm is very likely to correctly explain the kinematics of the TDGs.

\section{Data}

We use the HI rotation curves recently published by B07. They performed HI observations of the NGC 5291 system, using the VLA (Very Large Array) in its $\mathrm{BnA}$ and $\mathrm{CnB}$ configuration, yielding a spatial resolution of $\sim 2.2 \times 1.6 \mathrm{kpc}$. They discuss the ring structure composed of collisional debris that surrounds NGC 5291, and they detect eight clumps where velocity gradients can be observed. Only three of these, however, are resolved enough to allow a more detailed investigation of their kinematics.

The rotation curves of these three TDGs (NGC 5291N, NGC 5291S, and NGC 5291SW) were derived by B07 using an envelope-tracing method (Sancisi \& Allen 1979; Sofue 1996; Gentile et al. 2004), which gives reliable velocity estimates for poorly resolved and/or highly inclined disk galaxies. For the purpose of our analysis, we folded the two sides of the rotation curve $V_{\text {rot }}(r)$ and the baryonic contribution $V_{\text {bar }}(r)$, to obtain azimuthally averaged $V_{\text {rot }}(r)$ and $V_{\text {bar }}(r)$ curves. B07 use an inclination $i$ of $45^{\circ}$ for the three TDGs, based on the fact that their model reproduces successfully the morphology of the system with $i=45^{\circ}$, and that the rotation axis of the clumps differs from that of the ring by less than $15^{\circ}$. The total baryonic masses, as derived by B07, are: $9_{-0.7}^{+1.0} \times 10^{8} M_{\odot}$ for NGC $5291 \mathrm{~N}$, $9.3_{-0.9}^{+1.1} \times 10^{8} M_{\odot}$ for NGC 5291S, and $5 \pm 1.5 \times 10^{8} M_{\odot}$ for NGC 5291SW.

\section{Fitting procedure}

To make a rigorous fit of these galaxies in MOND, two important issues to take into account are their inclination and the EFE of MOND (e.g., Famaey et al. 2007; Wu et al. 2007; Angus \& McGaugh 2007). The rotation curves were thus fitted within the MOND framework with five different hypotheses:

- First, we simply applied the MOND prescription to the rotation curve. This approach has no explicit free parameter: we used $i$, the distance and the stellar mass-to-light $(M / L)$ ratio as derived by $\mathrm{B} 07$. The $M / L$ ratio is not a crucial assumption since the stellar mass, derived from stellar population synthesis models, is several times lower than the total baryonic mass. We used $a_{0}=1.2 \times 10^{-8} \mathrm{~cm} \mathrm{~s}^{-2}$ (Begeman et al. 1991).

- Second, we left $i$ as a free parameter. Indeed $i$ is an important parameter influencing the derived dynamical mass, and the inclination determined by B07 is only an estimate. The inclination we used is however constant with radius: i.e., we ignored any possible warps, which are taken into account in the error-bars.

- Third, we added a first estimate for the EFE, while $i$ was left as a free parameter. In modified gravity (Bekenstein \& Milgrom 1984), if one considers, as a first approximation, that a galaxy free-falls with a "uniform" acceleration in an external linear potential, the internal potential becomes polarized Keplerian (Milgrom 1986; Zhao \& Tian 2006; Zhao \& Famaey 2006). Here, to simplify the treatment of the EFE, we follow the less

\footnotetext{
1 A preliminary fit just posted on the arXiv by Milgrom (2007) has shown that MOND has the ability to explain the dynamics without resorting to disk dark matter.
}

rigorous approach of Famaey et al. (2007) in which the polarization in the direction of the external field (EF) as well as a term proportional to the gradient of $\mu$ are neglected. We define the internal acceleration as $a_{\text {int }}=V_{\text {obs }}^{2} / r$, where $V_{\text {obs }}$ is the observed rotation velocity and $r$ is the galactocentric radius in the TDGs. Then, following the MOND hypothesis: $a_{\text {int }}=g_{\mathrm{N}} / \mu(x)$, where $g_{\mathrm{N}}=V_{\mathrm{bar}}^{2} / r$ is the Newtonian acceleration, $\mu(x)=x /(1+x)$ (Famaey \& Binney 2005) and $x=\left(a_{\text {int }}+g_{\text {ext }}\right) / a_{0}$ (instead of the usual $x=a_{\text {int }} / a_{0}$ ), where $g_{\text {ext }}$ is the EF. We roughly estimate $g_{\text {ext }}$ due to NGC 5291 as follows: from the total HI profile $\Delta V_{20}$ (Malphrus et al. 1997), and assuming that the rotation curve of NGC 5291 stays flat out to the distances of the TDGs, we find $g_{\text {ext }} \lesssim\left(0.5 \Delta V_{20} / \sin (i)\right)^{2} / R_{\mathrm{G}}$, where $R_{\mathrm{G}}$ is the projected distance of the TDGs from the centre of NGC 5291. This gives $g_{\mathrm{ext}} \lesssim 0.3 a_{0}$. We then assume $g_{\mathrm{ext}}$ to have a typical value of $0.2 a_{0}$. Hence, the galaxies are mostly dominated by $a_{\text {int }}$ until the last data point, so the various approaches to take the EF into account are slightly degenerate, which we illustrate with $x=\max \left(a_{\mathrm{int}} / a_{0}, g_{\mathrm{ext}} / a_{0}\right)$ as a second parametrization. Our two choices of $x$ for the EFE bracket the range of possible effects of the $\mathrm{EF}$ on the rotation curve, the first one giving a larger EFE than the second. A more rigourous treatment of the effect of EF on rotation curves has already been done in $\mathrm{Wu}$ et al. (2007). It was found that the internal iso-potential contours are twisted due to the general orientations of the EF, but there are typically less than $10 \%$ difference among different orientations (hence the modulus of vector sums) of the EF and internal field. Rotation curves would become normal baryon-only Newtonian RCs if the system accelerates faster and faster in a stronger and stronger EF.

- Fourth, we fitted the rotation curves with Newtonian gravity and no dark matter, as would be expected in CDM, still keeping $i$ as a free parameter.

- Finally, we fixed $i$ at the most favourable inclination for the previous fits, and we derived the baryonic distribution required to fit the rotation curves in Newtonian gravity.

\section{Results}

In Fig. 1 we show the results of the fits. Overall MOND gives good fits to the rotation curves of these systems. In particular, Fig. 1 (panels a-c) shows that with no explicit free parameter, MOND can explain well the observed kinematics. As could be expected a priori, leaving $i$ as a free parameter improves the quality of the fits (see Fig. 1, panels d-f). The best-fit values are not far from the first assumption of $i=45^{\circ}$ : we find $i=50_{-7}^{+10} \mathrm{de}-$ grees for NGC $5291 \mathrm{~N}, i=34 \pm 4$ degrees for NGC 5291S, and $i=39_{-7}^{+11}$ degrees for NGC 5291SW: their average and scatter is similar to B07. It is interesting to note that all three TDGs fall on the baryonic Tully-Fisher (BTF) relation (McGaugh 2005): the agreement is perfect for $i$ as a free parameter and only slightly worse for $i=45^{\circ}$ (see Fig. 2). TDGs add to a long list of classes of galactic objects that satisfy the BTF relation. It is puzzling that an empirical relation works so well for galaxies of a wide range of formation histories unless it is the direct prediction of a realistic law of gravity relating to the instantaneous baryon distribution, as MOND is.

The fits with the two EFE estimates (see Fig. 1, panels g-i, for the first estimate) give good fits, whose best-fit parameters are displayed in Table 1. The first EFE estimate gives slightly worse fits than the second; we thus conclude that the EFE due to NGC 5291 in the 3 TDGs cannot be too large: it has to be smaller than $\sim(0.1-0.2) a_{0}$.

We realise that the EFE is a function of distance $R_{\mathrm{G}}$ from the host galaxy NGC 5291. The 3 TDGs are at projected distances 

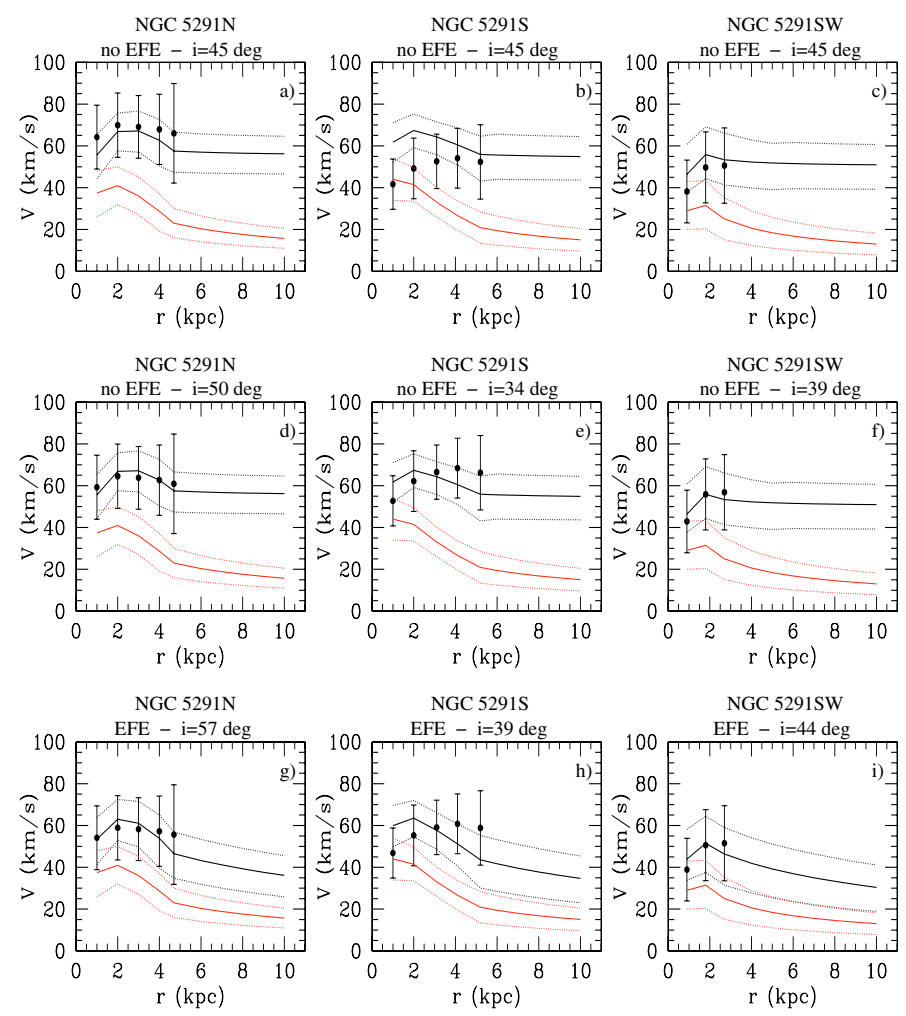

Fig. 1. Rotation curve data (full circles) of the 3 tidal dwarf galaxies (Bournaud et al. 2007). The lower (red) curves are the Newtonian contribution $V_{\mathrm{bar}}$ of the baryons (and its uncertainty, indicated as dotted lines). The upper (black) curves are the MOND prediction and its uncertainty (dotted lines). The top panels have as an implicit assumption (following Bournaud et al.) an inclination angle of 45 degrees. In the middle panels the inclination is a free parameter, and the bottom panels show the fits made with the first estimate for the external field effect (EFE) (Sect. 3).

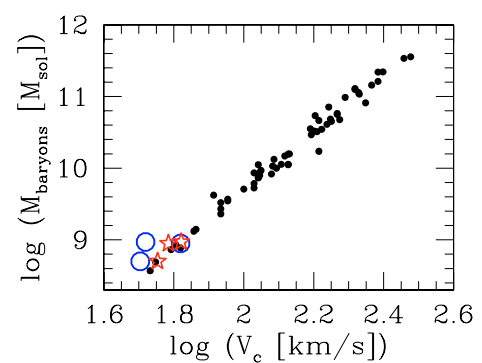

Fig. 2. Baryonic Tully-Fisher relation (baryonic mass vs. circular velocity). The small full circles are the disk galaxy data from McGaugh (2005). The 3 TDGs studied here are shown as blue empty circles $\left(i=45^{\circ}\right)$ and red stars $(i$ as a free parameter).

ranging from 58 to $75 \mathrm{kpc}$; given the small range of projected distances, and since the 3D distance is unknown, the EFE estimates are made using their average projected distance, $65 \mathrm{kpc}$, instead of their individual projected distances, which results in errors on the EFE $\lesssim 15 \%$, much smaller than the overall uncertainties on the actual EFE value. We ran models of the EFE with variations of $\sim 15 \%$, and found very similar results. Similarly, the difference between the EF effects on the two sides of the TDG rotation curves, is smaller than the uncertainty on its value, so we ignored it and worked on the azimuthally averaged rotation curve.

In Fig. 1 the curves with EFE show a Keplerian fall-off just outside the present data, a signal which is falsifiable if future
Table 1. Best-fit values of the 5 fits for each TDG. $i$ is the inclination, and the EFE estimates refer to Sect. 3. The 1- $\sigma$ uncertainties in the fitted inclinations are based on the $\chi^{2}$ statistics.

\begin{tabular}{cccc}
\hline \hline TDG name & $\chi^{2}$ & $i$ & Note \\
\hline NGC 5291N & 0.60 & $45 \mathrm{deg}$ & no EFE \\
& 0.16 & $50_{-71}^{+10} \mathrm{deg}$ & no EFE \\
& 0.30 & $57_{-9}^{+17} \mathrm{deg}$ & first EFE estimate \\
& 0.17 & $50_{-8}^{+10} \mathrm{deg}$ & second EFE estimate \\
& 3.60 & $90_{-16}^{+0} \mathrm{deg}$ & baryons + Newt. gravity \\
\hline NGC 5291S & 5.42 & $45 \mathrm{deg}$ & no EFE \\
& 1.36 & $34 \pm 4 \mathrm{deg}$ & no EFE \\
& 2.70 & $39_{-5}^{+6} \mathrm{deg}$ & first EFE estimate \\
& 1.70 & $35_{ \pm} 4 \mathrm{deg}$ & second EFE estimate \\
& 3.25 & $90_{-30}^{+0} \mathrm{deg}$ & baryons + Newt. gravity \\
\hline NGC 5291SW & 0.45 & $45 \mathrm{deg}$ & no EFE \\
& 0.09 & $39_{-7}^{+11} \mathrm{deg}$ & no EFE \\
& 0.19 & $44_{-9}^{+16} \mathrm{deg}$ & first EFE estimate \\
& 0.09 & $39_{-7}^{+11} \mathrm{deg}$ & second EFE estimate \\
& 0.42 & $90_{-33}^{+0} \mathrm{deg}$ & baryons + Newt. gravity \\
\hline
\end{tabular}

observations could extend the kinematics of these TDGs. This is not due to tidal truncation, but to the EFE. A rotating disk inclined with an angle $\theta_{\mathrm{EF}}$ with respect to the $\mathrm{EF}$ will generally precess around the axis of the EF (Wu et al. 2007; Famaey et al. 2007). The precession means that these TDG disks in MOND are not expected to be exactly aligned with the same inclinations, justifying our fits where $i$ is left as a free parameter. We note that continued accretion from the tidal matter and torquing of the TDGs may, however, affect the shape of the rotation curve at large radii, so the solid lines at large radii only show the case for unperturbed satellites.

We note that large uncertainties still exist in the mass distributions and inclinations of these TDGs, and thus in their dynamics. The scenario proposed by B07 within the CDM paradigm leaves some liberty as to the exact geometry and encounter. In particular, the inclination of the TDG on the sky planes could be more edge-on, and the inferred enclosed mass required to explain the observed velocities smaller, decreasing the need for dark matter or modified gravity. The overall quality of Newtonian, purely baryonic fits with $i=90^{\circ}$ and with the baryonic distribution taken from B07 (not shown in Fig. 1) is worse than the MOND fits with a free inclination, but it is only slightly worse than the MOND fits with $i=45^{\circ}$ : in NGC 5291N the MOND fit is much better, in NGC 5291S it is a bit worse, and in NGC 5291 the two fits are equivalent. However, given the geometry of the NGC 5291 system, it seems unlikely (but possible) that all 3 TDGs studied have such high inclinations. Also, the radial distribution of baryons, essentially the HI gas, is also poorly determined, and can be chosen to fit the observed rotation curves in Newtonian gravity, instead of being selected a priori. We have made such a fit (Fig. 3), by selecting exponential profiles for the surface density of the interstellar gas distributed in a disk. The enclosed gas mass within each radius is also plotted, together with the gas surface density, compatible with the observations. The deduced rotation curves are consistent with the data, within the error bars. Let us note that the fit is still rough, no effort was made to fit more components (such as the stellar component and the molecular gas) in addition to the HI gas.

Given the (relatively) poor quality of the rotation curves, a more rigorous study of the EFE and of the possible warps is difficult, since they are not well constrained observationally. The 

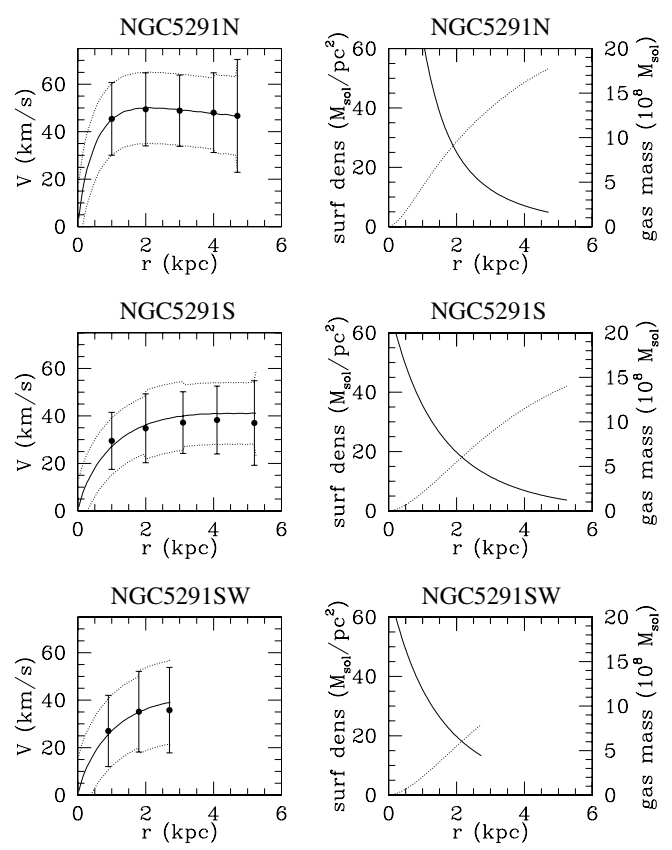

Fig. 3. Left: rotation curves with $i=90^{\circ}$, with the baryonic distribution that best fits them in Newtonian dynamics. Symbols and curves are like in Fig. 1. Right: the corresponding surface density distribution (solid line) and integrated mass (dotted line).

uncertainty on the EFE is unlikely to significantly affect our conclusions given that the EFE and the no-EFE results do not differ much.

\section{Conclusions}

We analysed the rotation curves of 3 TDGs presented by Bournaud et al. (2007) and we found that MOND explains their kinematics very naturally. Within the MOND framework, no additional unseen matter is required in these 3 TDGs. The dark matter required in Newtonian dynamics implies a large amount of unseen baryonic matter, which is very unconventional in the CDM context.

We explored different possibilities for the inclination angles and the external field effect (EFE) due to NGC 5291. The results do not change significantly: obviously the fits are improved with one extra free parameter (the inclination), and we find the external field to be smaller than $\sim(0.1-0.2) a_{0}$, within the a priori expectations from the kinematics of NGC 5291. Therefore, MOND can fit the kinematics of the 3 TDGs presented by Bournaud et al. (2007) for inclinations similar to those that they estimated and for realistic values of the external field.
We also found that in the (rather unlikely) case where all 3 TDGs are seen edge-on, Newtonian fits with no dark matter are possible but slightly worse than the MOND fits with $i=45^{\circ}$, and not as good as the MOND fits with $i$ as a free parameter. This Letter has thus shown that TDGs pose severe challenges to the current standard cosmological theory and that very recent observations of rotating TDGs are consistent with a non-Newtonian interpretation. TDGs thus hold important clues on fundamental physics and therewith require further research.

Acknowledgements. We thank S. McGaugh and G. Angus for useful suggestions, F. Bournaud for providing the kinematical data, and the anonymous referee for useful suggestions.

\section{References}

Angus, G. W., \& McGaugh, S. S. 2007, MNRAS, submitted [arXiv:0704.0381]

Barnes, J. E., \& Hernquist, L. 1992, Nature, 360, 715

Begeman, K. G., Broeils, A. H., \& Sanders, R. H. 1991, MNRAS, 249, 523

Bournaud, F., \& Duc, P.-A. 2006, A\&A, 456, 481

Bournaud, F., Duc, P.-A., Brinks, E., et al. 2007, Science, 316, 1166

Braine, J., Lisenfeld, U., Duc, P.-A., \& Leon, S. 2000, Nature, 403, 867

Calura, F., \& Matteucci, F. 2004, MNRAS, 350, 351

Delgado-Donate, E. J., Muñoz-Tuñón, C., Deeg, H. J., \& Iglesias-Páramo, J. 2003, A\&A, 402, 921

Duc, P.-A., \& Mirabel, I. F. 1998, A\&A, 333, 813

Duc, P.-A., Brinks, E., Springel, V., et al. 2000, AJ, 120, 1238

Famaey, B., \& Binney, J. 2005, MNRAS, 363, 603

Famaey, B., Bruneton, J.-P., \& Zhao, H. 2007, MNRAS, 377, L79

Gentile, G., Salucci, P., Klein, U., Vergani, D., \& Kalberla, P. 2004, MNRAS, 351,903

Gentile, G., Salucci, P., Klein, U., \& Granato, G. L. 2007, MNRAS, 375, 199

Łokas, E., Mamon, G., \& Prada, F. 2006, EAS Publ. Ser., 20, 113

McGaugh, S. S. 2005, ApJ, 632, 859

Metz, M., \& Kroupa, P. 2007, MNRAS, 376, 387

Milgrom, M. 1983, ApJ, 270, 365

Milgrom, M. 1986, ApJ, 302, 617

Milgrom, M., \& Sanders, R. H. 2003, ApJ, 599, L25

Milgrom, M. 2007, [arXiv:0706.0875]

Mirabel, I. F., Dottori, H., \& Lutz, D. 1992, A\&A, 256, L19

Monreal-Ibero, A., Colina, L., Arribas, S., \& Garcia-Marin, M. 2007, A\&A, 472, 421

Nipoti, C., Londrillo, P., \& Ciotti, L. 2007, [arXiv : 0705 .4633]

Okazaki, T., \& Taniguchi, Y. 2000, ApJ, 543, 149

Sancisi, R., \& Allen, R. J. 1979, A\&A, 74, 73

Sanders, R. H., \& McGaugh, S. S. 2002, ARA\&A, 40, 263

Sanders, R. H., \& Noordermeer, E. 2007, MNRAS, in press [arXiv: astro-ph/0703352]

Skillman, E. D., \& Bender, R. 1995, Rev. Mex. Astron. Astrof. Conf. Ser., 3, 25 Sofue, Y. 1996, ApJ, 458, 120

Weilbacher, P. M., Duc, P.-A., Fritze v. Alvensleben, U., Martin, P., \& Fricke, K. J. 2000, A\&A, 358, 819

Wu, X., Zhao, H. S., Famaey, B., et al. 2007 [arXiv: 0706. 3703]

Zhao, H. S., \& Famaey, B. 2006, ApJ, 638, L9

Zhao, H. S., \& Tian, L. 2006, A\&A, 450, 1005 